\title{
THE
}

\section{A sensitive method for the detection of legacy and emerging per- and polyfluorinated alkyl substances (PFAS) in dairy milk}

\author{
Nicholas I. Hill \\ University of Rhode Island \\ Jitka Becanova \\ University of Rhode Island \\ Rainer Lohmann \\ University of Rhode Island, rlohmann@uri.edu
}

Follow this and additional works at: https://digitalcommons.uri.edu/gsofacpubs

The University of Rhode Island Faculty have made this article openly available.

Please let us know how Open Access to this research benefits you.

This is a pre-publication author manuscript of the final, published article.

Terms of Use

This article is made available under the terms and conditions applicable towards Open Access

Policy Articles, as set forth in our Terms of Use.

\section{Citation/Publisher Attribution}

Hill, N.I., Becanova, J. \& Lohmann, R. A sensitive method for the detection of legacy and emerging perand polyfluorinated alkyl substances (PFAS) in dairy milk. Anal Bioanal Chem (2021). https://doi.org/ $10.1007 / \mathrm{s} 00216-021-03575-2$

Available at: https://doi.org/10.1007/s00216-021-03575-2

This Article is brought to you for free and open access by the Graduate School of Oceanography at DigitalCommons@URI. It has been accepted for inclusion in Graduate School of Oceanography Faculty Publications by an authorized administrator of DigitalCommons@URI. For more information, please contact digitalcommons-group@uri.edu. 
1 A sensitive method for the detection of legacy and emerging per- and

2 polyfluorinated alkyl substances (PFAS) in dairy milk

3 Nicholas Hill*, Jitka Becanova*\#, Rainer Lohmann*

4 *University of Rhode Island, Graduate School of Oceanography, Narragansett, Rhode Island,

5 USA

6 "corresponding author: Jitka Becanova, becanova@uri.edu

\section{Abstract}

8 There is widespread contamination by per- and polyfluoroalkyl substances (PFAS) across the

9 globe, with adverse effects on human and environmental health. For human exposure, drinking

10 water and dietary exposure have been recognized as important PFAS exposure pathway for the

11 general population. Several documented cases of dairy milk contamination by PFAS have

12 raised concerns over this exposure pathway in general. A sensitive method for determination of

1327 PFAS in milk was hence modified and applied on raw and processed milk samples from

14 thirteen farms across the United States (U.S.). A combination of acid and basic extraction

15 method and ENVI-Carb cleanup achieved recoveries of targeted PFAS between $70-141 \%$. The

16 method detection limits (MDL) ranged from $0.8-22 \mathrm{ng} / \mathrm{L}$ (for 26 PFAS) and $144 \mathrm{ng} / \mathrm{L}$ for

17 perfluorobutanoic acid (PFBA). The uniqueness of this method is considered in the targeted

18 screening of a broad range of legacy PFAS, as well as perfluorinated sulfonamide species and

19 fluorotelomer sulfonates. No legacy PFAS were detected in 13 milk samples from regions of

20 concern given local use of biosolids or proximity to fire training areas. Overall, then, the uptake

21 of perfluoroalkyl acids (PFAA) from dairy milk in the U.S. is considered low. 


\section{Graphical abstract}

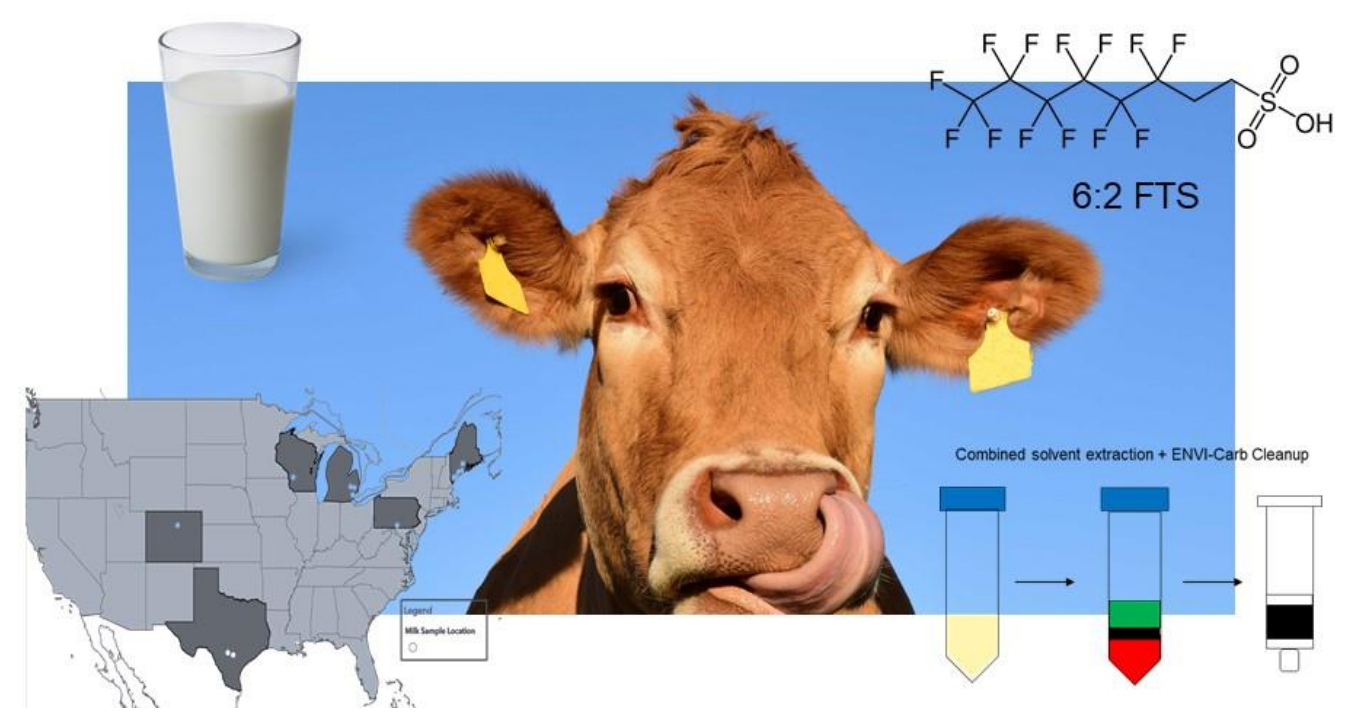

25 Keywords

26 AFFF, dairy milk, extraction method, FTS, PFAS

\section{Introduction}

28 Per- and polyfluoroalkyl substances (PFAS) comprise a broad group of anthropogenic

29 chemicals that are widely used in industrial and commercial applications [1]. These chemicals

30 display unique qualities such as lower micellization concentrations, ability to lower surface

31 tension of aqueous phases, hydrophobicity, and are oleophobic [2]. A variety of industries and

32 manufacturers have exploited these physicochemical properties to produce water repellent and

33 stain resistant coatings on textiles, oil-resistant food contact materials, and efficient aqueous

34 film forming foams (AFFF) [3]. As a result of their extensive use and chemical stability, PFAS

35 are ubiquitous in the environment and have been detected in wildlife and humans [4-6]. 
36 Extensive PFAS contamination in the environment has been predominantly linked to

37 applications of AFFF near airports, fire training areas, and military bases, as well as agricultural

38 use of biosolids or sludge derived from wastewater treatment plants (WWTP) [5,7-9]. Prolonged

39 applications of AFFF and WWTP biosolids and sludge are attributed to elevated PFAS

40 concentrations in soil and groundwater, as well as surface and well water [10-15]. At numerous

41 sites impacted by AFFF and biosolids, the concentrations of perfluorooctane sulfonate (PFOS)

42 and perfluorooctanoic acid (PFOA) in drinking water dramatically exceeded the U.S.

43 Environmental Protection Agency (EPA) lifetime health advisory level of $70 \mathrm{ng} / \mathrm{L}$ for the

44 combined concentration of these two compounds [16-18].

45 Ingestion of such as contaminated drinking water is a significant human exposure pathway in

46 addition to PFAS ingested through diet $[19,20]$. In general, human dietary PFAS exposure

47 occurs by two main routes: i) direct exposure to PFAS present in unprocessed, raw products as

48 a result of environmental contamination, and ii) indirect exposure to PFAS present in food

49 contact materials used in manufacturing, packaging, and preparation of processed food [21].

50 Dietary PFAS exposure pathways and PFAS contribution vary for different populations [21]. For

51 instance, the European Food Safety Authority (EFSA) estimated that fish and seafood are

52 predominant pathways for chronic PFAS exposure in adults to PFOS (up to $86 \%$ ). The EFSA

53 also projected that milk and dairy products are significant PFAS chronic exposure pathways to

54 vulnerable populations (e.g., toddlers) [20]. The PFAS contamination in milk and dairy products

55 could originate from processing and packaging of the final products, but most likely comes from

56 transfer of PFAS from feed to cows. This was previously demonstrated in both a dosing study

57 [22] and a descriptive model [23] in which dairy milk became a reservoir for PFAS. With a

58 continuous increase in annual milk production in the U.S. over the last decade [24], there is

59 plausible concern for increased risk of dietary exposure to PFAS to the general U.S population. 
60 While a range of retail food studies including raw milk and other dairy products have been

61 conducted in both farm and local market products all over the world [21,25-30] a limited number

62 of reports exists for the U.S. domestic food supply. Previous studies have demonstrated that

63 livestock forage grown on biosolid-amended soils is an important driver of PFAS contamination

64 of the cattle [15,31]. Similarly, the use of organic fertilizers mixed with industrial wastes on

65 cropland has led to contamination of the cattle feedlots and subsequently to elevated PFAS

66 concentrations in animal by-products such as meat [22]. In multiple studies, the cattle exposed

67 to contaminated feed eliminate PFAS via lactational transfer [15,22,32]. Application of

68 contaminated biosolids has also been documented in farms across the U.S. for which PFAS

69 concentrations reached up to thousands of micrograms per kilogram of biosolids $[15,31,33]$.

70 Therefore, agricultural application of WWTP biosolids or industrial wastes or the proximity of

71 dairy farms to AFFF-impacted areas warrants concern for PFAA contamination in dairy

72 production.

73 Previous research on PFAS contamination of dairy cow milk has placed a greater emphasis on

74 limited number of legacy perfluorinated alkyl acids (PFAA) with a focus on PFOA and PFOS

$75[21,22,25,28-30,34,35]$. Elevated contamination in milk was mostly attributed to the ability of

76 PFAA to bind to $\beta$-lactoglobulin proteins in cow milk $[26,36]$ however limited data exists on both

77 milk concentrations and mechanism of binding/releasing of emerging PFAS associated with

78 AFFF and WWTP biosolids applications such as polyfluorinated fluorotelomer sulfonates,

79 perfluoroalkyl sulfonamidoacetic acids, and perfluoroalkyl sulfonamides [17,37].

80 Previously developed extraction methods for PFAS analysis in milk used a small volume of the

81 samples $(1-5 \mathrm{~mL})$ to minimize the lipid and protein content in the final extracts and prevent

82 potential matrix effect during the instrumental analysis [38-40]. Additionally, up-to-date

83 published milk extraction method targeted the legacy PFAA only, without including the novel

84 PFAA alternatives and precursors $[21,22,25,26,28-30,35]$. The aim of the present study was 
85 therefore to i) modify solvent digestion and sample cleanup method for broader group of legacy, 86 emerging and precursor PFAS in raw dairy cow milk and ii) apply the extraction method on raw 87 dairy milk collected from U.S. dairy farms.

\section{Materials and methods}

\section{Standards and reagents}

90 The 8-point calibration curve $(0.004-100 \mathrm{ng} / \mathrm{mL}), \mathrm{QA} / \mathrm{QC}$ instrumental performance check, and

91 surrogate standard were created using analytical PFAS standards purchased from Wellington

92 Laboratories (Ontario, Canada). Individual target PFAS and corresponding isotope labelled

93 analogues are listed in Table SI 1. Formic acid (99+\%), ammonium hydroxide (28\%-30\%), and

94 liquid chromatography-mass spectrometry (LC-MS) grade methanol were purchased from

95 Fisher Scientific (Pittsburgh, PA, USA). Oasis WAX solid-phase extraction resin (30 $\mu \mathrm{m})$ was

96 purchased from Waters (Milford, MA, USA) and ENVI-Carb cartridges (Supelco) were

97 purchased from Sigma-Aldrich.

\section{Extraction Method Evaluation}

99 To evaluate the efficiency of extraction methods, approximately $10 \mathrm{~g}$ of local market, whole-milk 100 was weighed into wide-mouth polypropylene jars $(n=9)$, spiked with representative native 101 PFAS standards ( $10 \mathrm{ng} / \mathrm{sample}$ ) and placed overnight in a freezer at $-15^{\circ} \mathrm{C}$ before freeze drying 102 at $-53^{\circ} \mathrm{C}$ for $\sim 60$ hours in a LABCONCO FreeZone $2.5 \mathrm{~L}$ benchtop freeze dry system. Freeze 103 dried milk ( $\sim 2 \mathrm{~g}$ ) was transferred to $15 \mathrm{~mL}$ Corning $\AA$ Falcon centrifuge tubes and divided into 104 treatment groups ( $n=3$ per treatment) based on extraction solvent and clean-up procedure: i)

$1050.1 \%$ formic acid in methanol followed by clean-up with only ENVI-Carb cartridges, ii) $0.1 \%$ 106 formic acid in methanol extraction followed by clean-up with Oasis WAX resin loaded atop of 107 ENVI-Carb cartridges, and iii) $0.1 \%$ ammonium hydroxide in methanol followed by clean-up with 108 Oasis WAX powder loaded atop of ENVI-Carb cartridges. To create the paired WAX resin with 
ENVI-Carb cartridge, approximately $500 \mathrm{mg}$ of WAX resin suspended in LC-MS-grade methanol

110 was transferred onto the Envi-Carb cartridge $(1 \mathrm{~g})$ with a pre-cleaned disposable pipette.

111 To extract PFAS from the milk matrix, $6 \mathrm{~mL}$ of solvent was added to samples according to

112 respective treatment groups. All samples were then vortexed for $\sim 30 \mathrm{~s}$, placed in an ultrasonic

113 bath for $20 \mathrm{~min}$, and centrifuged at $4000 \mathrm{rpm}$ for $10 \mathrm{~min}$. ENVI-Carb cartridges were fixed to a

114 CHROMABOND SPE manifold. All cartridges were pre-cleaned prior to sample loading with $~ 3$

$115 \mathrm{~mL}$ each of $0.1 \%$ formic acid in methanol, $0.1 \%$ ammonium hydroxide in methanol, and finally

116 neutral methanol. The organic layer was then transferred with a disposable transfer pipette and

117 loaded onto the cartridges. $15 \mathrm{~mL}$ Falcon centrifuge tubes were placed inside the manifold to

118 capture milk extracts. Samples were allowed to elute under gravity. The pellet formed during

119 centrifugation was rinsed with $\sim 2 \mathrm{~mL}$ of neutral methanol and resuspended, vortexed $\sim 30 \mathrm{~s}$,

120 placed in an ultrasonic bath for $20 \mathrm{~min}$, and centrifuged at $4000 \mathrm{rpm}$ for $10 \mathrm{~min}$. The organic

121 layer was then transferred as detailed above onto the respective ENVI-Carb cartridge after the

122 first extract eluted. After gravity elution of the last fraction, a final wash of $\sim 1-2 \mathrm{~mL}$ of neutral

123 methanol was used to rinse the inside of the cartridge. Vacuum pressure $(<15$ psi) was applied

124 to the manifold to remove residual solvent extract bound within the cartridges. Approximately 9-

$12510 \mathrm{~mL}$ of extraction eluent was present after SPE clean-up procedures. This eluent was

126 evaporated at $36^{\circ} \mathrm{C}$ to $\sim 0.5 \mathrm{~mL}$ under a gentle stream of nitrogen gas and spiked with mass

127 labeled PFAS mix (2 ng/sample). $40 \mu \mathrm{L}$ of the concentrated extract was diluted with $60 \mu \mathrm{L}$ of 4

$128 \mathrm{mM}$ ammonium acetate in water prior to LC-MS analysis.

\section{Sample collection and storage}

130 Milk samples $(n=13)$ were collected from 13 individual cattle farms across The United States

131 (Table SI 2, Figure 1). These dairy cattle farms reported use of biosolid amendments on

132 cropland or were located within proximity to AFFF-impacted soils. Samples were shipped on ice

133 in original storage containers. All sample storage containers, extraction vessels, and transfer 
134 pipettes were pre-cleaned with ACS-grade methanol, 3\% ammonium hydroxide in LC-MS-grade 135 methanol, and LC-MS-grade methanol prior to use. Thawed milk samples were partitioned into 136 pre-cleaned $1 \mathrm{~L} \mathrm{HDPE}$ bottles for storage at $-15^{\circ} \mathrm{C}$. Locally purchased pasteurized whole milk 137 was used for determinations of dairy matrix interference with instrument detection. Samples 138 were analyzed within their shelf lives. A representative summary of sample collection locations 139 can be found in Table SI 2.

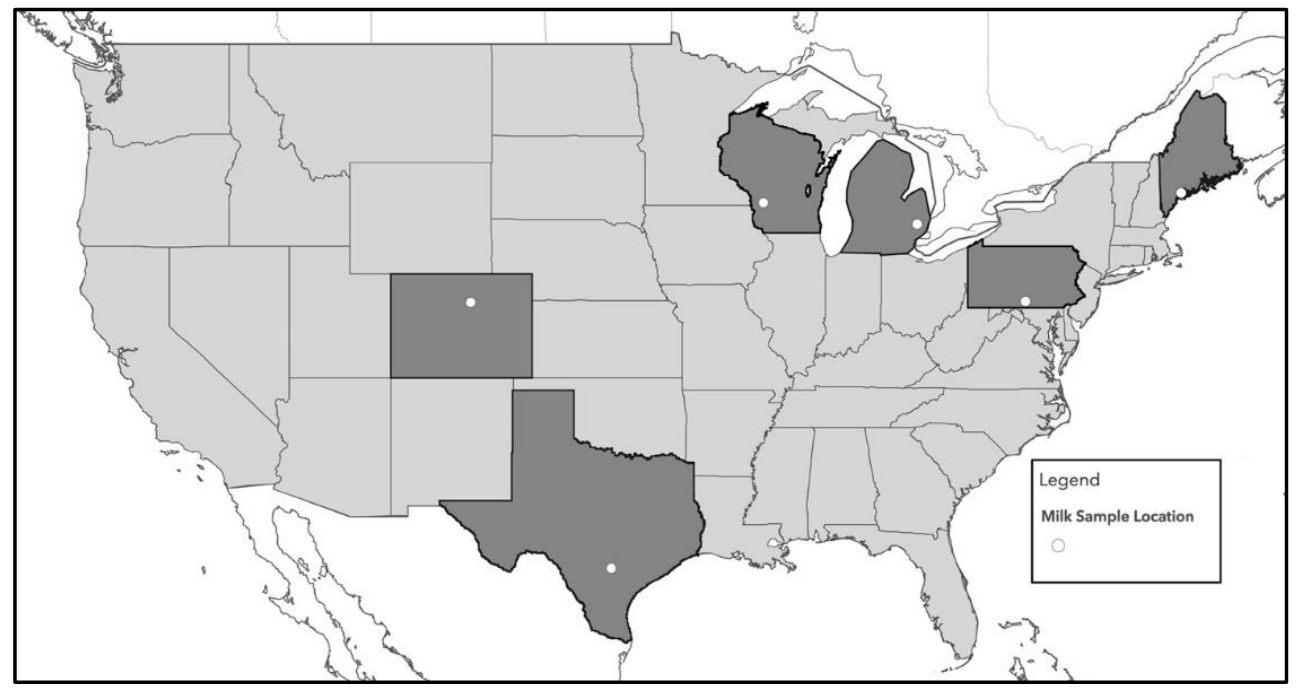

Figure 1: Map of milk samples location in selected states (Colorado, Maine, Michigan, Pennsylvania, Texas and Wisconsin) in The United States

\section{Sample preparation}

144 Frozen milk samples were allowed to thaw at room temperature and well mixed before $\sim 25 \mathrm{~g}$ of

145 thawed samples were weighed into pre-cleaned $50-\mathrm{mL}$ polypropylene Corning $\AA^{\circledR}$ Falcon

146 centrifuge tubes. All samples, duplicates, matrix spikes and blanks were spiked with mass

147 labeled surrogate PFAS standard mixture (4 ng/sample). Additionally, a native PFAS solution (4 $148 \mathrm{ng} / \mathrm{sample}$ ) was added to matrix spike milk samples. Sample aliquots were frozen overnight at -

$14915{ }^{\circ} \mathrm{C}$, followed by $-80^{\circ} \mathrm{C}$ for five hours the next day, before freeze-drying in a LABCONCO ${ }^{8}$

150 FreeZone2.5 for $60 \mathrm{~h}$ at $-54^{\circ} \mathrm{C}$. After freeze-drying, sample extraction was conducted using a 151 combined solvent digestion procedure. 
152 Briefly, $12 \mathrm{~mL}$ of $0.1 \%$ formic acid in LC-MS-grade methanol was added to each freeze-dried 153 milk sample to denature proteins. Samples were then vortexed for $\sim 30 \mathrm{~s}$ and placed in an ultra154 sonic bath for $25 \mathrm{~min}$ at room temperature before centrifugation at $4000 \mathrm{rpm}$ for $10 \mathrm{~min}$. The 155 organic supernatant was then transferred to a $15 \mathrm{~mL}$ Corning® Falcon centrifuge tube and 156 concentrated down to $\sim 1 \mathrm{~mL}$ under a gentle stream of nitrogen gas to allow room for additional 157 aliquots. Following the initial concentration step, $6 \mathrm{~mL}$ of LC-MS-grade methanol was added to 158 the original pellet formed in the first solvent digestion step. The same vortex, sonication, and 159 centrifugation steps were repeated. After centrifugation, the organic supernatant was transferred 160 and combined with the concentrated acidic digestion extract. Lastly, a final solvent digestion 161 was performed with $6 \mathrm{~mL}$ of $0.1 \%$ ammonium hydroxide in methanol following the same 162 procedures as outlined in the previous solvent digestions. A final sample concentration under a 163 gentle stream of nitrogen gas down to $\sim 4 \mathrm{~mL}$ was performed. The final volume extracts were 164 stored overnight at $-15^{\circ} \mathrm{C}$ to promote precipitation of residual matrix within extracts.

165 The sample clean-up procedure was performed with ENVI-carb (1 g, Supelco) cartridges. 166 Cartridges were affixed to a CHROMABOND® SPE manifold and precleaned with $2 \mathrm{~mL}$ each of $1670.1 \%$ formic acid in methanol, $0.1 \%$ ammonium hydroxide in methanol, and lastly LC-MS-grade 168 methanol. Prior to loading, samples were taken out of freezer storage, centrifuged to remove 169 residual matrix for $1 \mathrm{~min}$ at $4000 \mathrm{rpm}$. The supernatant was then transferred into ENVI-carb 170 cartridges and allowed to elute under gravity ( 1 drop/sec) into fresh $15 \mathrm{~mL}$ Corning® Falcon 171 centrifuge tubes. A $1 \mathrm{~mL}$ wash with LC-MS-grade methanol was performed on the original 172 storage tube and centrifugation for $1 \mathrm{~min}$ at $4000 \mathrm{rpm}$ conducted prior to loading this extract to 173 the cartridge. Additionally, cartridges were rinsed with a final $1 \mathrm{~mL}$ LC-MS-grade methanol 174 aliquot. Lastly, vacuum pressure ( 10 psi) was applied to elute residual solvent extract bound 175 within the cartridge. Eluents were concentrated down to $\sim 0.5 \mathrm{~mL}$ under a gentle stream of 176 nitrogen gas before preparation for HPLC-MS/MS analysis. 


\section{Instrumental LC-MS analysis}

178 The LC-MS/MS analysis of targeted PFAS (Table SI 1) was performed using a liquid

179 chromatograph (Shimadzu Prominence UFLC) equipped with a Gemini C18 hybrid column (3

$180 \mu \mathrm{m}, 2.1 \mathrm{~mm} \times 50 \mathrm{~mm}$; Phenomenex) coupled to mass spectrometer (AB Sciex 4500 QTRAP)

181 operating in negative ion mode. To reduce background contamination in the system, a delay

182 column (Luna $5 \mu \mathrm{m} \mathrm{C18(2)} 100 \AA$, LC Column $30 \times 2 \mathrm{~mm}$ ) was installed to the LC system. For

183 analysis, $20 \mu \mathrm{L}$ of prepared extract was injected on the analytical column and PFAS were

184 separate and determined (all analytical details are listed in SI, Table SI 3, 4 and 5 and in [41]).

\section{QA/QC}

186 The calculations of the PFAS concentration in samples and quality control samples was based

187 on the isotope dilution method of quantitation. To guarantee quality control, three process

188 blanks and two matrix spikes blanks were included within each batch of 14 samples. Blank

189 concentrations were $<10 \%$ of the measured samples, and due to this low background

190 contamination level, sample concentrations were not blank corrected. The method detection

191 limits (MDL, ng/L) were determined considering the following criteria: in case no analyte signal

192 was detected in the process blanks, instrumental detection limits (IDL) were used as MDL and

193 an appropriate dilution factor was applied. IDL represents the concentration of analyte giving the

194 signal-to-noise ratio of 10 in presence of the matrix. In case the analytes were detected in

195 process blanks, MDL were calculated as average value plus 3 times the standard deviation (SD)

196 of the concentrations in all blanks. MDLs and recoveries for all targeted PFAS are listed in Table

1971 (with details in SI). Additionally, recoveries of the surrogate mass labeled PFAS spiked into

198 the real samples, blanks and quality control samples were withing $60-140 \%$. 


\section{Results and discussion}

\section{Extraction Method Evaluation}

201 The extraction method (solid liquid extraction, SLE) evaluation incorporated nine retail milk

202 replicates for which two different extraction solvents and two different clean-up methods were

203 utilized. When extracting PFAS from milk, it is common to incorporate solvents or salts to 204 denature and precipitate proteins and other biochemical artifacts that may bind PFAS, such a $\beta$

205 -lactoglobulin [25,26,28]. For these reasons, a similar approach was applied, relying on either $2060.1 \%$ formic acid (FA) in methanol (treatment 1 and 2 ) or $0.1 \%$ ammonium hydroxide $(\mathrm{AH})$ in 207 methanol (treatment 3).

208 A summary of 22 native PFAS recoveries from extraction is provided in Figure 2. Average 209 recoveries for the C4-C10 PFCAs was generally over 60\% between treatments (Fig. 2a).

210 Among the C4-C11 PFCAs, incorporation of 500 mg WAX powder loaded atop of the ENVI-

211 Carb cartridge $(1 \mathrm{~g})$ did not result in significantly higher recoveries. Recoveries of the C12-C14

212 PFCAs were generally over 50\% except for PFTeDA (treatment 2). Average recoveries of C4

213 and C6-C8 PFSAs (Fig. 2b) ranged from 48\% to 51\% with basic digestion (treatment 3)

214 extraction being most optimal for the recovery of the sulfonates. PFBS had the greatest 215 recovery of the PFSAs at $63 \%$. Recovery of the sulfonamides (Fig. 2c): FBSA, FOSA, $n$ 216 MeFOSA, and $n$-EtFOSA ranged from $23 \%$ to $71 \%$ across experiments with recovery of FOSA 217 being the highest at $71 \%$ in Treatment 1 . The recoveries for $n$-MeFOSA, and $n$-EtFOSA were 218 overall low, so these compounds were excluded from further evaluations. Recoveries for the 219 fluorotelomer sulfonates (Fig. 2d) ranged from $42 \%$ to $68 \%$, with highest average recovery 220 across treatment groups residing with 6:2 FTS at 68\%, and lowest average recovery with 4:2 221 FTS at $42 \%$. Generally, the target PFAA and polyfluorinated precursors had recoveries of $\sim 60 \%$ 222 on average. The WAX powder allowed for greater separation of C4-C12 PFCA, PFBS, PFHxS, 223 PFHpS, PFOS, and other perfluorinated species from the interfering matrix. However, the 
224 recoveries for longer chain PFCA which are known for their bioaccumulative properties [42]

225 were higher on average for Treatment 3 which incorporated use of $0.1 \%$ ammonium hydroxide

226 in methanol. The recoveries of PFCA were generally higher in Treatment 3 when compared to

227 Treatment 1. A similar pattern can be seen with the PFSA and 6:2 FTS. Slight differences were

228 evident in the recovery for PFOS between Treatment 1 and Treatment 3 (Fig. 2b). These

229 patterns provided justification to utilize a stepwise solvent extraction which incorporates both

230 acidic and basic organic solvents to account for the broad spectrum of predominant PFAS found

231 in WWTP wastewater and biosolids, as well as AFFF [14,37,43-45]. Therefore, the combination

232 of the treatment 1 and 3 was applied on the real milk samples to achieve maximum recoveries

233 for all targeted group of PFAS. 


\section{a) PFCA}

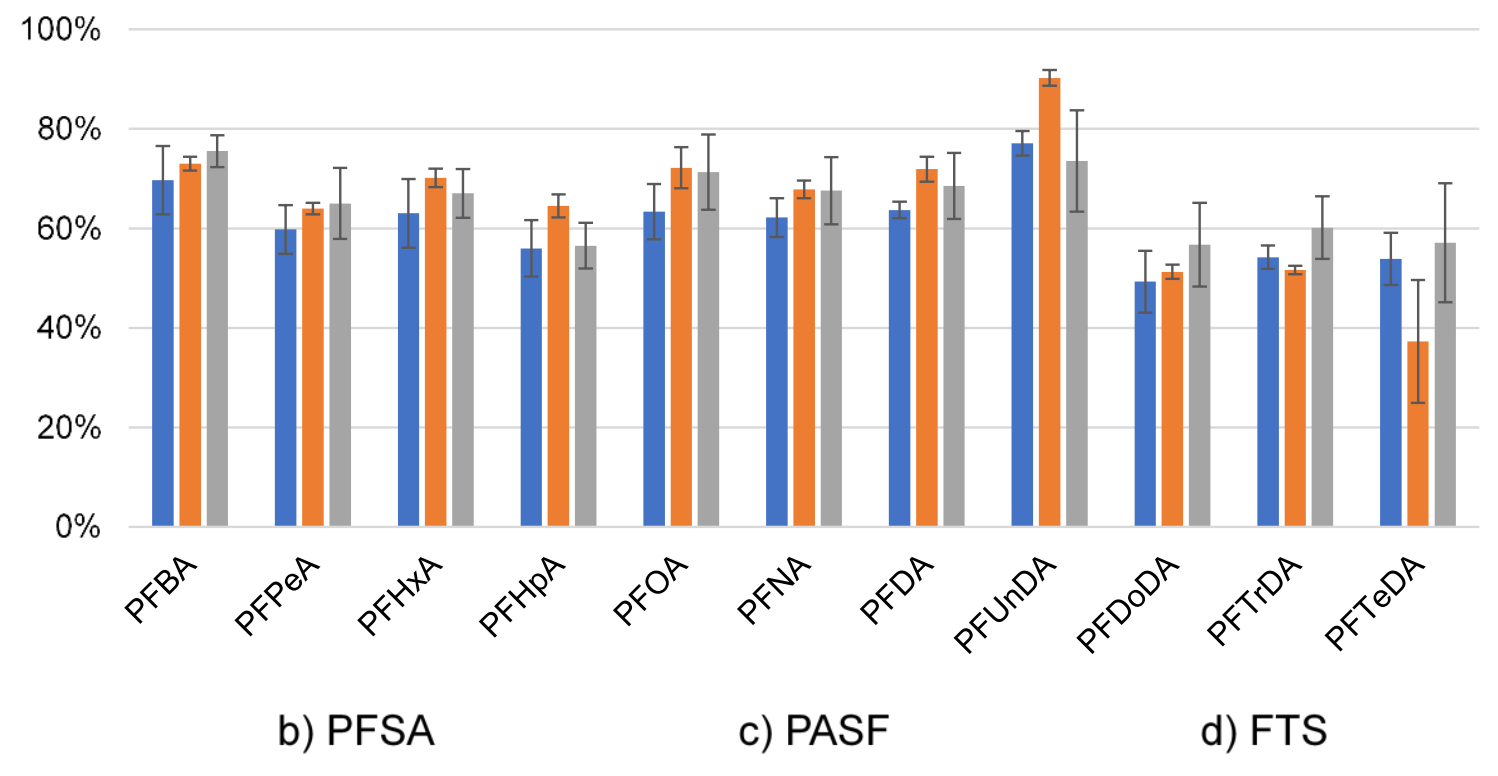

$100 \%$

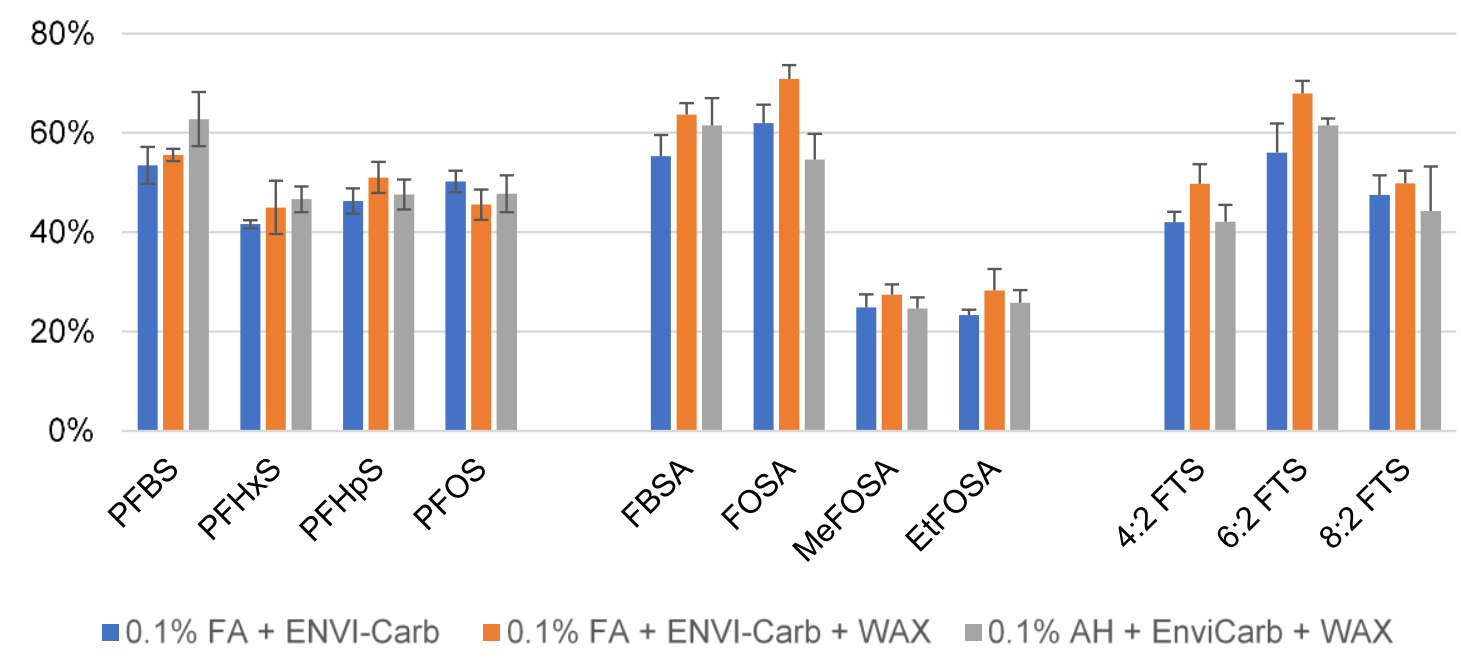

Figure 2: Recoveries of individual per- (1a, 1b, and 1c) poly- (1c) fluorinated compounds using three different treatment methods. 1a-PFCA (perfluorocarboxylic acids); $1 b-P F S A$ (perfluorosulfonic acids); 1c-PASF based compounds (perfluoro sulfonamides); and 1d - FTS (fluorotelomer sulfonates). Treatments $(n=3)$ : i) $0.1 \%$ FA in methanol + clean-up with ENVI-Carb (blue), ii) $0.1 \%$ FA in methanol + clean-up with Oasis WAX loaded atop of ENVI-Carb (orange), and iii) $0.1 \%$ AH in methanol + clean-up with Oasis WAX loaded atop of ENVI-Carb (grey) 


\section{Analysis of real milk samples}

243 A total of thirteen raw and retail milk samples were collected from U.S. dairy farms that either

244 had confirmed use of biosolids on cropland or were within geographic proximity to military

245 installations with confirmed AFFF use. PFAS present in AFFF utilized at fire training areas and

246 military bases may persist in soils and groundwater leachate $[17,46,47]$. PFAS recalcitrance in

247 soils due to AFFF leachate irrigation or biosolid amendment application pose reasonable

248 concerns for agriculture [48]. Concentration of PFOA and PFOS in plants grown in biosolid

249 amended soils have previously been found up to $200 \mathrm{ng} / \mathrm{g} \mathrm{dw}$ and $20 \mathrm{ng} / \mathrm{g} \mathrm{dw}$, respectively [49].

250 PFOS concentrations in biosolids from previous studies found as little as 4.3 to $89 \mu \mathrm{g} / \mathrm{kg} \mathrm{dw} \mathrm{[50]}$

251 to as much as $3120 \mu \mathrm{g} / \mathrm{kg}$ in the U.S. [31], reaching elevated concentrations that have

252 ubiquitous concern for biosolid use in agriculture. Where WWTP biosolids have been spread on

253 cropland, PFOS concentrations have been quantified up to $483 \mu \mathrm{g} / \mathrm{kg} \mathrm{dw}[33,51]$ took into

254 consideration WWTP biosolid amendments and the likely occurrence of biotransfer from crop to

255 organism by providing toxicokinetic evidence of PFOA uptake and elimination in beef cattle.

256 Following this, Kowalczyk et al. (2013) demonstrated elimination of PFAS from naturally

257 contaminated feed, in part, through lactational transfer [22]. Accumulation of PFAS in animal by-

258 products therefore serves as a possible endpoint for exposure to humans who incorporate dairy

259 milk in their diet. Both the proximity of farms to AFFF-impacted soils and the presence of WWTP

260 biosolids on croplands raises concerns for bioaccumulation in food animals whose feed is

261 obtained from the cropland.

262 A targeted LC-MS analysis of 27 PFAS (Table SI 1) was conducted on the raw and retail milk

263 samples for which a combined solvent extraction and ENVI-Carb clean-up was performed. The

264 efficiency of the modified solvent digestion extraction and clean-up procedure is summarized in

265 Table 1. The overall recoveries of the 27 PFAS were evaluated by using a real milk samples

266 spiked with native PFAS solution (4ng per sample). The recoveries were calculated using the 
267 isotope dilution method. Recoveries for the 27 targeted analytes ranged from $69 \pm 9 \%$ to $141 \pm$

$2685 \%$. The average recovery amongst the PFAA (13 compounds) was $93 \%$, similar to recoveries

269 for PFCA with $\mathrm{CF}_{2} \leq 10$ previously determined in other studies $[21,26,28]$, which ranged from 70

270 to $120 \%$. Only Lacina et al. [26] demonstrated similar performance for longer chain PFCA using

271 a multistep ion pair extraction and cleanup method. Within the group of PFAS (8 compounds)

272 the method achieved an average recovery of $113 \%$, ranging from $90 \pm 6 \%$ to $141 \pm 5 \%$, the

273 lowest being PFECHS and PFNS as the highest, respectively. In above mentioned studies the

274 smaller range of PFSA (3 to 5) was dominantly analyzed with recoveries ranging from 70 to

$275104 \%$. For the 4 sulfonamides and sulfonamide acids, the average recoveries were $97 \%$.

276 Recovery for the only previously determined sulfonamide from this group (FOSA) was $107 \pm$

$2771 \%$ which is comparable to previously published recoveries $98 \%$ [26]. Lastly, recoveries of the

278 fluorotelomer sulfonates ( 3 compounds) ranged from $98 \pm 3 \%$ to $136 \pm 7 \%$, with lowest recovery

279 of 6:2 FTS and highest recovery of 8:2 FTS, respectively. 
281 Table 1: Calculated Recoveries (\% $\pm S D$ ) and Method Detection Limits (MDLs) for analysis of 282 real samples

\begin{tabular}{|c|c|c|c|c|c|}
\hline $\begin{array}{c}\text { Functional } \\
\text { group }\end{array}$ & Fluorination & $\mathrm{n}\left(\mathrm{CF}_{2}\right)$ & Compound & $\begin{array}{c}\text { Recovery }(\%) \pm \\
S D\end{array}$ & MDL (ng/L) \\
\hline \multirow{11}{*}{$\begin{array}{c}1 \\
-\mathrm{COOH}\end{array}$} & \multirow{11}{*}{ Per- } & 4 & PFBA & $69 \pm 9$ & 144 \\
\hline & & 5 & PFPeA & $90 \pm 0$ & 7.6 \\
\hline & & 6 & PFHxA & $91 \pm 3$ & 3.9 \\
\hline & & 7 & PFHpA & $120 \pm 0$ & 11.0 \\
\hline & & 8 & PFOA & $82 \pm 0$ & 8.8 \\
\hline & & 9 & PFNA & $84 \pm 9$ & 2.2 \\
\hline & & 10 & PFDA & $86 \pm 13$ & 1.6 \\
\hline & & 11 & PFUnDA & $118 \pm 17$ & 3.6 \\
\hline & & 12 & PFDoDA & $110 \pm 17$ & 5.7 \\
\hline & & 13 & PFTrDA & $91 \pm 14$ & 5.3 \\
\hline & & 14 & PFTeDA & $88 \pm 5$ & 2.8 \\
\hline \multirow{8}{*}{$\begin{array}{c}2 \\
-\mathrm{SO}_{3} \mathrm{H}\end{array}$} & \multirow{8}{*}{ Per- } & 4 & PFBS & $117 \pm 8$ & 22 \\
\hline & & 5 & PFPeS & $94 \pm 8$ & 3.6 \\
\hline & & 6 & PFHxS & $106 \pm 4$ & 11 \\
\hline & & 7 & PFHpS & $103 \pm 1$ & 11 \\
\hline & & 8 & PFOS & $112 \pm 6$ & 2.9 \\
\hline & & 8 & PFECHS & $90 \pm 6$ & 2.3 \\
\hline & & 9 & PFNS & $141 \pm 5$ & 12.9 \\
\hline & & 10 & PFDS & $115 \pm 8$ & 2.4 \\
\hline \multirow{5}{*}{$\begin{array}{c}3 \\
-\mathrm{SO}_{2} \mathrm{~N}\end{array}$} & \multirow{5}{*}{ Per- } & 4 & FBSA & $105 \pm 8$ & 1.9 \\
\hline & & 6 & $\mathrm{FH} \times \mathrm{SA}$ & $80 \pm 12$ & 0.8 \\
\hline & & 8 & FOSA & $107 \pm 1$ & 5.2 \\
\hline & & 8 & MeFOSAA & $112 \pm 8$ & 2.1 \\
\hline & & 8 & EtFOSAA & $81 \pm 1$ & 1.4 \\
\hline \multirow{3}{*}{$\begin{array}{c}4 \\
-\mathrm{SO}_{3} \mathrm{H}\end{array}$} & \multirow{3}{*}{ Poly- } & 4 & 4:2 FTS & $105 \pm 8$ & 1.9 \\
\hline & & 6 & 6:2 FTS & $98 \pm 3$ & 1.6 \\
\hline & & 8 & 8:2 FTS & $136 \pm 7$ & 2.0 \\
\hline
\end{tabular}


284 We evaluated the method performance on the real samples and calculated the method 285 detection limits (MDLs) for the SLE-HPLC-MS/MS as described above. Generally, MDL ranged 286 from $0.8-22 \mathrm{ng} / \mathrm{L}$ for 26 PFAS and $144 \mathrm{ng} / \mathrm{L}$ for PFBA, which is known for a strong matrix 287 interference. Achieved MDL are far below the only established action level for PFAS (PFOS;

$288210 \mathrm{ng} / \mathrm{L}$ ) in cow's milk developed by Maine Department of Agriculture, Conservation and 289 Forestry (DACF) the Maine Center for Disease Control and Prevention (MECDC) [52].

290 The uniqueness of this method is considered in the targeted screening of a broad range of 291 legacy PFAS, as well as perfluorinated sulfonamide species and fluorotelomer sulfonates, for 292 which MDL $<5.2 \mathrm{ng} / \mathrm{L}$ were achieved. To our knowledge, this is one of the first studies to screen 293 such a variety of legacy and emerging PFAS in the U.S. produced milk.

294 The stepwise solvent digestion method incorporating the use of acidic, and basic methanolic 295 solvents for initial extraction of PFAS in cow milk is the first of its kind in the literature together 296 with a condensed clean-up to a single ENVI-Carb cartridge to help remove milk sample matrix. 297 This improved clean-up and extraction method achieved recoveries that were as good as or 298 better for target PFAS in comparison to other dairy cow milk studies where MDLs varied 299 between hundreds $\mathrm{pg} / \mathrm{L}$ to tens $\mathrm{ng} / \mathrm{L}$ for limited number of PFAA $[21,22,25,26,28-30,35]$. 
301 Table 2: Comparison of concentration ( $\mathrm{ng} / \mathrm{L})$ of various group of PFAS in dairy milk samples

\begin{tabular}{|c|c|c|c|c|c|c|}
\hline \multirow{2}{*}{ Country } & \multirow{2}{*}{$\mathbf{n}$} & \multicolumn{4}{|c|}{ Concentration range (min-max) $\mathrm{ng} / \mathrm{L}$} & \multirow{2}{*}{ Reference } \\
\hline & & PFCA & PFSA & PASF & FTS & \\
\hline $\begin{array}{l}\text { The Czech } \\
\text { Republic }\end{array}$ & 12 & $<\mathrm{MDL}$ & $<\mathrm{MDL}$ & $<M D L$ & $N A^{*}$ & [26] \\
\hline USA & 61 & NA & $<M D L-0.16$ & NA & NA & [21] \\
\hline Italy & 15 & $<M D L$ & $<M D L$ & NA & NA & [30] \\
\hline Germany & 14 & $<M D L-10.1$ & $<M D L-8.5$ & NA & NA & [28] \\
\hline $\begin{array}{l}\text { The } \\
\text { Netherlands }\end{array}$ & 17 & $<\mathrm{MDL}$ & $<\mathrm{MDL}$ & NA & NA & [53] \\
\hline Italy & 67 & $<\mathrm{MDL}-32$ & $<M D L-97$ & NA & NA & [29] \\
\hline China & 46 & $<M D L-370$ & $<M D L-120$ & NA & NA & [34] \\
\hline China & 115 & $<M D L-151.8$ & $<M D L-172.9$ & NA & NA & [35] \\
\hline Taiwan & 10 & $30-1440$ & $<\mathrm{MDL}-10$ & NA & NA & [25] \\
\hline USA & 13 & $<\mathrm{MDL}$ & $<\mathrm{MDL}$ & $<M D L$ & $<\mathrm{MDL}-6.59$ & this study \\
\hline
\end{tabular}

$302{ }^{*} N A-$ not analyzed in the particular study

303 The present study included milk samples that were collected from a variety of rural dairy farms

304 that sell to local markets and larger urban areas (Table SI 1, Figure 1). The only analyte

305 detected in this study was 6:2 FTS at concentration $6.6 \mathrm{ng} / \mathrm{L}$, for which a lack of data is

306 available from previous studies regarding contamination in dairy cow milk (Table 2). To our

307 knowledge, the only other U.S.-based study (Table 2) similarly investigated biosolid-amended

308 croplands and concerns for accumulation of PFAS in dairy milk and quantified only PFOS (0.16

$309 \mathrm{ng} / \mathrm{L})$ above its MDL (0.13 ng/L) [21]. Both 6:2 FTS and PFOS can commonly be found in both

310 AFFF leachate and WWTP biosolids [10,44]. With PFAS concentrations in most milk samples

311 being below their MDLs, and a representative number of dairy farms and locations included in

312 this study, the data suggests that consumption of dairy milk is not a prominent source of dietary

313 PFAS exposure. Similarly, proximity to military zones with historical AFFF use does not seem to 
314 be a factor. However, as recent evidence suggests, the presence of biosolids containing PFAS

315 may lead to contamination in soils and plants on cropland [15]. Especially short chain PFAA

316 such as PFBA, PFPeA and PFBS are well known to be accumulated by agriculture plants

$317[54,55]$ but it is unknown as to whether the cattle on these farms frequently graze on cropland

318 associated with biosolid spreading or if these short chain PFAA which show different elimination

319 kinetics compare to the long chain PFAA due to smaller molecular size have been excreted via

320 urine [22].

\section{Conclusion}

322 The method presented in this study demonstrated enhanced capacity to quantitatively analyzed 323 a broad range of PFAS in dairy milk in sub $\mathrm{ng} / \mathrm{L}$ using a combined solvent extraction and single

324 step clean-up procedure. Using this method, we screened raw and processed milk samples

325 from dairy cattle farms which reported use of biosolid amendments on cropland or were located

326 within proximity to AFFF-impacted soils. While levels of legacy PFAS formerly known to

327 accumulate in a variety of dairy products were below detection limits, the fluorotelomer sulfonate

328 (6:2 FTS) was detected in one sample. These findings might reflect shifts in the AFFF

329 compositions thus the further exploration of PFAS contamination of dairy products using non-

330 targeted screening or total extractable fluorine approach might be essential.

\section{Acknowledgments}

332 The authors acknowledge funding from the US National Institute of Environmental Health

333 Sciences (grant P42ES027706); and Richard J. Valdmanis and Joshua S. Schneyer (Reuters

334 journalists) for collecting milk samples at local markets in six states. 


\section{Author information}

336 Affiliations

337 University of Rhode Island, Graduate School of Oceanography, Narragansett, Rhode Island, 338 USA

339 Nicholas Hill, Jitka Becanova, Rainer Lohmann

340 Corresponding author

341 Correspondence to Jitka Becanova, becanova@uri.edu

342

\section{Ethics declarations}

\section{Conflict of interest}

345 The authors declare no competing interests. 


\section{References}

348 [1] Kissa E. Fluorinated surfactants and repellents. vol. 607. New York: Marcel Dekker Inc.; 3492001.

350 [2] Moody CA, Field JA. Perfluorinated Surfactants and the Environmental Implications of Their Use in Fire-Fighting Foams. Environ Sci Technol 2000;34:3864. https://doi.org/10.1021/es991359u.

[3] Glüge J, Scheringer M, Cousins IT, DeWitt JC, Goldenman G, Herzke D, et al. An overview of the uses of per-and polyfluoroalkyl substances (PFAS). EngrXiv 2020;8:Web.

[4] Lau C, Anitole K, Hodes C, Lai D, Pfahles-Hutchens A, Seed J. Perfluoroalkyl acids: a review of monitoring and toxicological findings. Toxicol Sci 2007;99:366-94.

[5] Schulz K, Silva MR, Klaper R. Distribution and effects of branched versus linear isomers of PFOA, PFOS, and PFHxS: A review of recent literature. Sci Total Environ 2020;733. https://doi.org/10.1016/j.scitotenv.2020.139186.

[6] De Silva AO, Armitage JM, Bruton TA, Dassuncao C, Heiger-Bernays W, Hu XC, et al. PFAS Exposure Pathways for Humans and Wildlife: A Synthesis of Current Knowledge and Key Gaps in Understanding. Environ Toxicol Chem 2021;40:631-57. https://doi.org/https://doi.org/10.1002/etc.4935.

[7] Filipovic M, Woldegiorgis A, Norström K, Bibi M, Lindberg M, Österås AH. Historical usage of aqueous film forming foam: A case study of the widespread distribution of perfluoroalkyl acids from a military airport to groundwater, lakes, soils and fish. Chemosphere 2015;129. https://doi.org/10.1016/j.chemosphere.2014.09.005.

[8] Hu XDC, Andrews DQ, Lindstrom AB, Bruton TA, Schaider LA, Grandjean P, et al. Detection of Poly- and Perfluoroalkyl Substances (PFASs) in US Drinking Water Linked to Industrial Sites, Military Fire Training Areas, and Wastewater Treatment Plants. Environ Sci Technol Lett 2016;3:344-50. 
372 [9] Semerád J, Hatasová N, Grasserová A, Černá T, Filipová A, Hanč A, et al. Screening for 32 per- and polyfluoroalkyl substances (PFAS) including GenX in sludges from 43 WWTPs located in the Czech Republic - Evaluation of potential accumulation in vegetables after application of biosolids. Chemosphere 2020;261:128018. https://doi.org/https://doi.org/10.1016/j.chemosphere.2020.128018.

377 [10] Schultz MM, Barofsky DF, Field JA. Quantitative determination of fluorotelomer sulfonates in groundwater by LC MS/MS. Environ Sci Technol 2004;38:1828-35.

379 [11] Kärrman A, Elgh-Dalgren K, Lafossas C, Møskeland T. Environmental levels and distribution of structural isomers of perfluoroalkyl acids after aqueous fire-fighting foam (AFFF) contamination. Environ Chem 2011;8:372-80.

[12] F. Houtz E, P. Higgins C, A. Field J, L. Sedlak D. Persistence of Perfluoroalkyl Acid Precursors in AFFF-Impacted Groundwater and Soil. Environ Sci \&amp; Technol 2013;47:8187-95. https://doi.org/10.1021/es4018877.

[13] Houtz EF, Sutton R, Park JS, Sedlak M. Poly- and perfluoroalkyl substances in wastewater: Significance of unknown precursors, manufacturing shifts, and likely AFFF impacts. Water Res 2016;95. https://doi.org/10.1016/j.watres.2016.02.055.

[14] Clarke BO, Smith SR. Review of 'emerging' organic contaminants in biosolids and assessment of international research priorities for the agricultural use of biosolids. Environ Int 2011;37:226-47. https://doi.org/https://doi.org/10.1016/j.envint.2010.06.004.

[16] Filipovic M, Berger U. Are perfluoroalkyl acids in waste water treatment plant effluents the result of primary emissions from the technosphere or of environmental recirculation? Chemosphere 2015;129:74-80. https://doi.org/http://dx.doi.org/10.1016/j.chemosphere.2014.07.082. 
[17] Weber AK, Barber LB, LeBlanc DR, Sunderland EM, Vecitis CD. Geochemical and Hydrologic Factors Controlling Subsurface Transport of Poly- and Perfluoroalkyl Substances, Cape Cod, Massachusetts. Environ Sci Technol 2017;51:4269-79. https://doi.org/10.1021/acs.est.6b05573.

[18] Lindstrom AB, Strynar MJ, Delinsky AD, Nakayama SF, McMillan L, Libelo EL, et al. Application of WWTP Biosolids and Resulting Perfluorinated Compound Contamination of Surface and Well Water in Decatur, Alabama, USA. Environ Sci Technol 2011;45:801521. https://doi.org/Doi 10.1021/Es1039425.

[19] Domingo JL. Health risks of dietary exposure to perfluorinated compounds. Environ Int 2012;40:187-95.

[20] Sunderland EM, Hu XC, Dassuncao C, Tokranov AK, Wagner CC, Allen JG. A review of

[21] Young WM, South P, Begley TH, Diachenko GW, Noonan GO. Determination of Perfluorochemicals in Cow's Milk Using Liquid Chromatography-Tandem Mass Spectrometry. J Agric Food Chem 2012;60:1652-8. https://doi.org/10.1021/jf204565x.

[22] Kowalczyk J, Ehlers S, Oberhausen A, Tischer M, Fürst P, Schafft H, et al. Absorption, Distribution, and Milk Secretion of the Perfluoroalkyl Acids PFBS, PFHxS, PFOS, and PFOA by Dairy Cows Fed Naturally Contaminated Feed. J Agric Food Chem 2013;61:2903-12. https://doi.org/10.1021/jf304680j.

[23] van Asselt ED, Kowalczyk J, van Eijkeren JCH, Zeilmaker MJ, Ehlers S, Fürst P, et al. Transfer of perfluorooctane sulfonic acid (PFOS) from contaminated feed to dairy milk. Food Chem 2013;141:1489-95. https://doi.org/https://doi.org/10.1016/j.foodchem.2013.04.035.

[24] USDA. Milk production. Natl Agric Stat Serv n.d. https://doi.org/ISSN: 1949-1557. 
424 [25] Chen W-L, Bai F-Y, Chang Y-C, Chen P-C, Chen C-Y. Concentrations of perfluoroalkyl substances in foods and the dietary exposure among Taiwan general population and pregnant women. J Food Drug Anal 2018;26:994-1004. https://doi.org/10.1016/j.jfda.2017.12.011.

[26] Lacina O, Hradkova P, Pulkrabova J, Hajslova J. Simple, high throughput ultra-high performance liquid chromatography/tandem mass spectrometry trace analysis of perfluorinated alkylated substances in food of animal origin: Milk and fish. J Chromatogr A 2011;1218:4312-21. https://doi.org/10.1016/j.chroma.2011.04.061.

Domingo JL, Jogsten IE, Eriksson U, Martorell I, Perelló G, Nadal M, et al. Human dietary exposure to perfluoroalkyl substances in Catalonia, Spain. Temporal trend. Food Chem 2012;135:1575-82. https://doi.org/10.1016/j.foodchem.2012.06.054.

[28] Still M, Schlummer M, Gruber L, Fiedler D, Wolz G. Impact of Industrial Production and

[29] Barbarossa A, Gazzotti T, Zironi E, Serraino A, pagliuca G. <em>Short Packaging Processes on the Concentration of Per- and Polyfluorinated Compounds in Milk and Dairy Products. J Agric Food Chem 2013;61:9052-62. https://doi.org/10.1021/jf4020137. communication:</em> Monitoring the presence of perfluoroalkyl substances in Italian cow milk. J Dairy Sci 2014;97:3339-43. https://doi.org/10.3168/jds.2014-8005.

[30] Capriotti AL, Cavaliere C, Cavazzini A, Foglia P, Laganà A, Piovesana S, et al. High performance liquid chromatography tandem mass spectrometry determination of perfluorinated acids in cow milk. J Chromatogr A 2013;1319:72-9. https://doi.org/10.1016/j.chroma.2013.10.029.

[31] Lupton SJ, Huwe JK, Smith DJ, Dearfield KL, Johnston JJ. Distribution and Excretion of Perfluorooctane Sulfonate (PFOS) in Beef Cattle (Bos taurus). J Agric Food Chem 2014;62:1167-73. https://doi.org/10.1021/jf404355b.

[32] Kowalczyk J, Ehlers S, Fürst P, Schafft H, Lahrssen-Wiederholt M. Transfer of 
Perfluorooctanoic Acid (PFOA) and Perfluorooctane Sulfonate (PFOS) From

Contaminated Feed Into Milk and Meat of Sheep: Pilot Study. Arch Env Contam Toxicol 2012;63:288-98. https://doi.org/10.1007/s00244-012-9759-2.

[33] Lupton SJ, Huwe JK, Smith DJ, Dearfield KL, Johnston JJ. Absorption and Excretion of 14C-Perfluorooctanoic Acid (PFOA) in Angus Cattle (Bos taurus). J Agric Food Chem 2012;60:1128-34. https://doi.org/10.1021/jf2042505.

[34] Yu Y, Xu D, Lu M, Zhou S, Peng T, Yue Z, et al. QuEChERs Combined with Online Interference Trapping LC-MS/MS Method for the Simultaneous Determination of 20 Polyfluoroalkane Substances in Dietary Milk. J Agric Food Chem 2015;63:4087-95. https://doi.org/10.1021/acs.jafc.5b00068.

[35] Xing Z, Lu J, Liu Z, Li S, Wang G, Wang X. Occurrence of Perfluorooctanoic Acid and

[36] Macheka LR, Olowoyo JO, Mugivhisa LL, Abafe OA. Determination and assessment of human dietary intake of per and polyfluoroalkyl substances in retail dairy milk and infant Perfluorooctane Sulfonate in Milk and Yogurt and Their Risk Assessment. Int J Environ Res Public Heal 2016;13. https://doi.org/10.3390/ijerph13101037. formula from South Africa. Sci Total Env 2021;755:142697.

https://doi.org/10.1016/j.scitotenv.2020.142697.

[37] Moodie D, Coggan T, Berry K, Kolobaric A, Fernandes M, Lee E, et al. Legacy and emerging per- and polyfluoroalkyl substances (PFASs) in Australian biosolids. Chemosphere 2021;270:129143. https://doi.org/https://doi.org/10.1016/j.chemosphere.2020.129143.

[38] Keller JM, Calafat AM, Kato K, Ellefson ME, Reagen WK, Strynar M, et al. Determination of perfluorinated alkyl acid concentrations in human serum and milk standard reference materials. Anal Bioanal Chem 2010;397:439-51. https://doi.org/10.1007/s00216-009-

475 [39] Tittlemier SA, Braekevelt E. Analysis of polyfluorinated compounds in foods. Anal Bioanal 
Chem 2011;399:221-7. https://doi.org/10.1007/s00216-010-4112-y.

477 [40] Li F, Zhao Z, Shen C, Zeng Q, Liu S. Elimination of matrix effects during analysis of perfluorinated acids in solid samples by liquid chromatography tandem mass spectrometry. J Cent South Univ 2012;19:2886-94. https://doi.org/10.1007/s11771-0121355-0.

[41] Becanova J, Saleeba Z, Stone A, Hurt RH, Robuck AR, Lohmann R. A graphene-based hydrogel monolith with tailored surface chemistry for PFAS passive sampling. Environ Sci

[42] $\mathrm{Ng} \mathrm{CA}$, Hungerbuhler K, Hungerbühler K. Bioaccumulation of Perfluorinated Alkyl Acids: Observations and Models. Environ Sci Technol 2014;48:4637-48. https://doi.org/10.1021/es404008g.

[43] Oono S, Harada KH, Mahmoud MAM, Inoue K, Koizumi A. Current levels of airborne polyfluorinated telomers in Japan. Chemosphere 2008;73:932-7.

[44] Coggan TL, Moodie D, Kolobaric A, Szabo D, Shimeta J, Crosbie ND, et al. An investigation into per- and polyfluoroalkyl substances (PFAS) in nineteen Australian wastewater treatment plants (WWTPs). Heliyon 2019;5:e02316. https://doi.org/https://doi.org/10.1016/j.heliyon.2019.e02316.

[45] Alder AC, van der Voet J. Occurrence and point source characterization of perfluoroalkyl acids in sewage sludge. Chemosphere 2015;129:62-73. https://doi.org/https://doi.org/10.1016/j.chemosphere.2014.07.045.

[46] Anderson RH, Long GC, Porter RC, Anderson JK. Occurrence of select perfluoroalkyl substances at U.S. Air Force aqueous film-forming foam release sites other than firetraining areas: Field-validation of critical fate and transport properties. Chemosphere 2016;150:678-85. https://doi.org/10.1016/J.CHEMOSPHERE.2016.01.014.

500 [47] Nickerson A, Rodowa AE, Adamson DT, Field JA, Kulkarni PR, Kornuc JJ, et al. Spatial 501 Trends of Anionic, Zwitterionic, and Cationic PFASs at an AFFF-Impacted Site. Environ 
Sci Technol 2021;55:313-23. https://doi.org/10.1021/acs.est.0c04473.

503 [48] Costello MCS, Lee LS. Sources, Fate, and Plant Uptake in Agricultural Systems of Perand Polyfluoroalkyl Substances. Curr Pollut Reports 2020. https://doi.org/10.1007/s40726-020-00168-y.

[49] Yoo H, Washington JW, Jenkins TM, Ellington JJ. Quantitative determination of perfluorochemicals and fluorotelomer alcohols in plants from biosolid-amended fields using LC/MS/MS and GC/MS. Environ Sci Technol 2011;45:7985-90.

[50] Brambilla G, Abate V, Battacone G, De Filippis SP, Esposito M, Esposito V, et al.

Potential impact on food safety and food security from persistent organic pollutants in top soil improvers on Mediterranean pasture. Sci Total Environ 2016;543:581-90. https://doi.org/https://doi.org/10.1016/j.scitotenv.2015.10.159.

[51] Sepulvado JG, Blaine AC, Hundal LS, Higgins CP. Occurrence and Fate of Perfluorochemicals in Soil Following the Land Application of Municipal Biosolids. Environ Sci Technol 2011;45:8106-12. https://doi.org/10.1021/es103903d.

[52] MECDC. Action levels for PFOS in cow's milk. Memorandum to the Department of Agriculture, Conservation and Forestry from the Maine CDC 2017.

[53] Berendsen BJA, Lakraoui F, Leenders L, van Leeuwen SPJ. The analysis of perfluoroalkyl substances at ppt level in milk and egg using UHPLC-MS/MS. Food Addit Contam Part A 2020;37:1707-18. https://doi.org/10.1080/19440049.2020.1794053.

[54] Blaine AC, Rich CD, Hundal LS, Lau C, Mills MA, Harris KM, et al. Uptake of Perfluoroalkyl Acids into Edible Crops via Land Applied Biosolids: Field and Greenhouse Studies. Environ Sci Technol 2013;47:14062-9. https://doi.org/10.1021/es403094q.

[55] Krippner J, Falk S, Brunn H, Georgii S, Schubert S, Stahl T. Accumulation Potentials of Perfluoroalkyl Carboxylic Acids (PFCAs) and Perfluoroalkyl Sulfonic Acids (PFSAs) in Maize (Zea mays). J Agric Food Chem 2015;63:3646-53. 
528 https://doi.org/10.1021/acs.jafc.5b00012.

529 